\title{
A Patient with a Delayed Diagnosis of Case Report Artery of Percheron Occlusion in Whom Thrombectomy Was Effective
}

Mitsuhiro Iwasaki, ${ }_{1}$ Chiyoe Hikita, ${ }^{1}$ Masahiro Maeda, ${ }^{1}$ Yasufumi Inaka, ${ }^{1}$ Hidekazu Yamazaki, ${ }^{2}$ Shinya Fukuta, ${ }^{1}$ Hiroaki Sato, ${ }^{1}$ and Masafumi Morimoto ${ }^{1}$

Objective: A case of posterior cerebral artery (P1 segment) occlusion with consciousness disturbance and Weber's syndrome treated by mechanical thrombectomy is reported.

Case Presentation: The patient was a 69-year-old man with consciousness disturbance, left hemiparesis, and anisocoria. MRI revealed acute cerebral infarction in the midbrain and right thalamus. Angiography demonstrated that the right P1 segment was occluded and mechanical thrombectomy was performed. The right P1 segment and its perforator artery, the artery of Percheron (AOP), were both recanalized after the treatment, and the symptoms of perforator occlusion significantly improved.

Conclusion: Mechanical thrombectomy for P1 segment occlusion may be effective for improving the symptoms caused by occlusion of its perforator, the AOP.

Keywords > Percheron artery, posterior cerebral artery, ischemic stroke, thrombectomy, perforator infarction

\section{Introduction}

Acute-phase thrombectomy is the standard treatment for large vessel occlusion (LVO) of the anterior circulation. Following extension of the time limit for its application, ${ }^{1,2)}$ and recognition of its effectiveness in the posterior circulation $^{3)}$ and peripheral vessles, ${ }^{4}$ the number of cases treated by this method is increasing. If perforator branches arise from a large vessel, infarction may develop in the area supplied by the perforators due to embolic LVO, but this can be improved by recanalization of the large vessel. ${ }^{5}$

We report a patient who presented with consciousness disturbance and Weber's syndrome due to occlusion of the

${ }^{1}$ Department of Neurosurgery, Yokohama Shintoshi Neurosurgical Hospital, Yokohama, Kanagawa, Japan

${ }^{2}$ Department of Neurology, Yokohama Shintoshi Neurosurgical Hospital, Yokohama, Kanagawa, Japan

Received: July 15, 2020; Accepted: January 27, 2021 Corresponding author: Mitsuhiro Iwasaki. Department of Neurosurgery, Yokohama Shintoshi Neurosurgical Hospital, 433, Edacho, Aoba-ku, Yokohama, Kanagawa 225-0013, Japan Email: imitsu1209@gmail.com

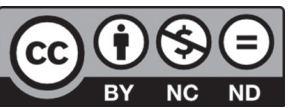

This work is licensed under a Creative Commons Attribution-NonCommercial-

NoDerivatives International License.

(C)2021 The Japanese Society for Neuroendovascular Therapy artery of Percheron (AOP) branching from the P1 segment of the right posterior cerebral artery, and had a favorable outcome after retrieval of the thrombus in the right $\mathrm{P} 1$ with a review of the literature.

\section{Case Presentation}

The patient was a 69-year-old man with a history of lip cancer and psoriasis. He previously underwent radiation therapy for lip cancer. On the day of the onset, the patient was found with consciousness disturbance in a car that stopped after a traffic accident and was emergently transported to our hospital. On arrival, the Japan Coma Scale (JCS) score was 3 , and anisocoria with the diameters of the right and left pupils were 4 and $2 \mathrm{~mm}$, respectively, and left hemiparesis were observed. The National Institutes of Health Stroke Scale (NIHSS) score was 9. No high-intensity areas were observed on diffusion weighted image (DWI) by MRI (Fig. 1A), and the left internal carotid artery and from the end of the basilar artery (BA) to the P1 segments of the bilateral posterior cerebral arteries were poor (Fig. 1B), but the $\mathrm{P} 2$ and more distal areas were clearly shown (Fig. 1C). Although no fresh infarction was noted in the images, we suspected fresh infarction near the brainstem based on the symptoms and MRA findings, and admitted the patient. However, after admission, his condition deteriorated to JCS20 and 


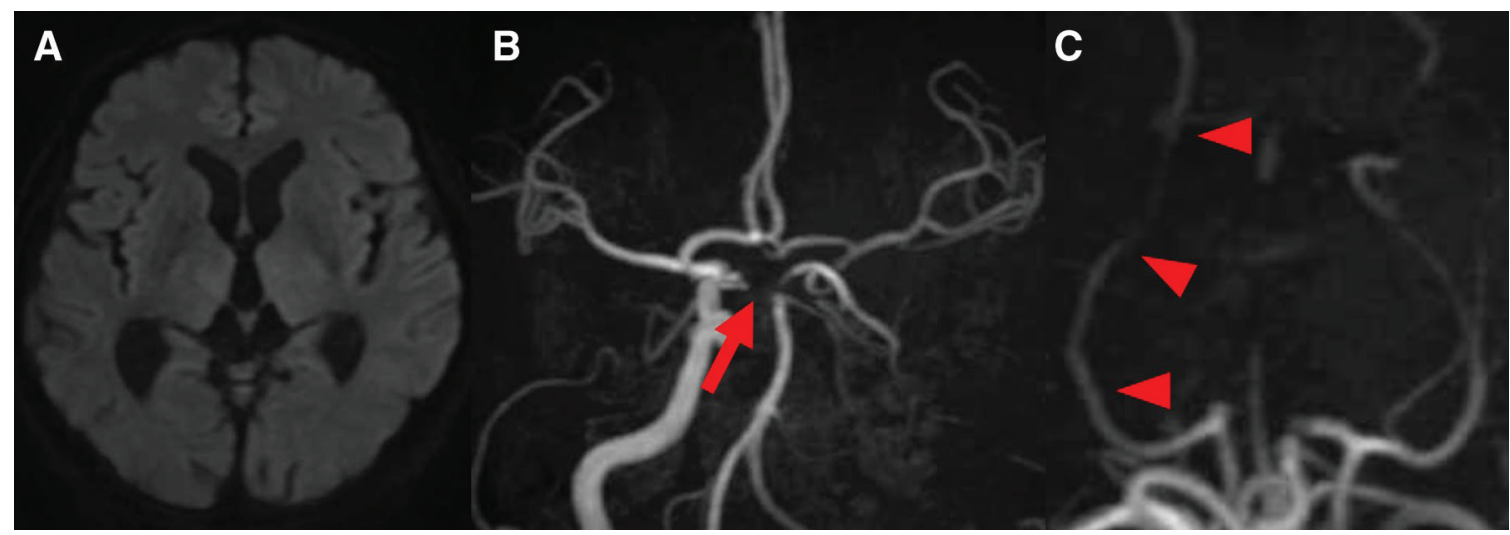

Fig. $1 \mathrm{MRI}$ and MRA on admission. (A) DWI shows no acute cerebral infarction. (B) MRA shows occlusion of the left internal carotid artery and occlusion of the bilateral P1 segments (arrow). (C) MRA shows the right posterior cerebral artery (arrowheads) with the developed posterior communicating artery. DWI: diffusion weighted image

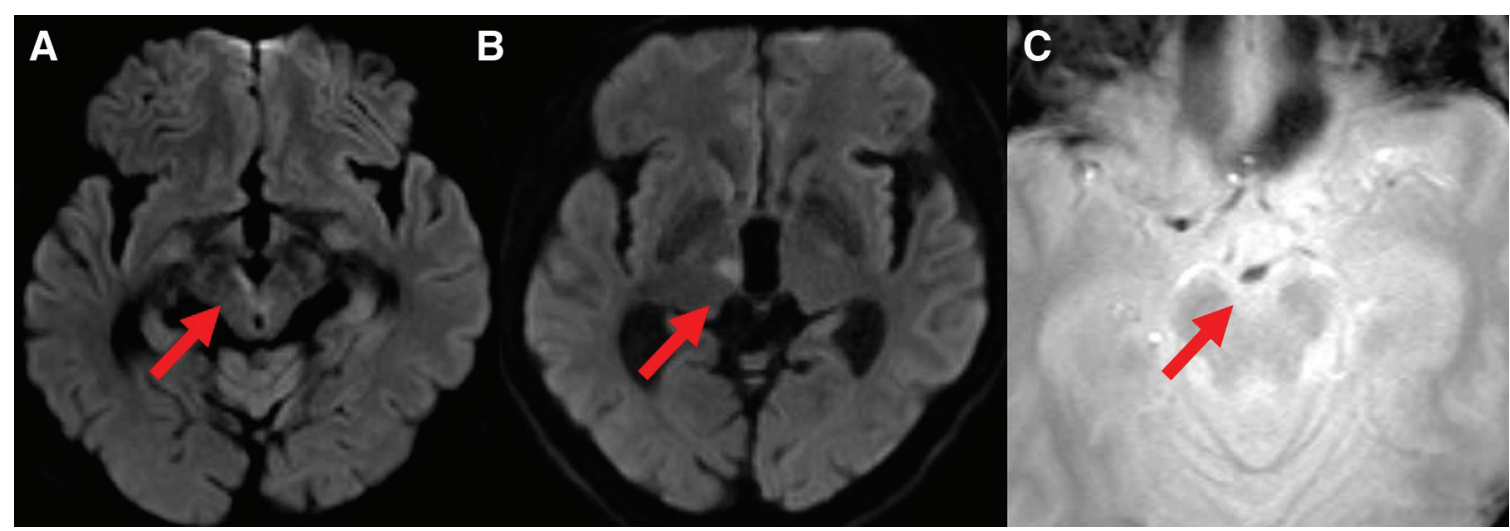

Fig. 2 Preoperative MRI DWI shows hyper-intense signals in the right cerebral peduncle (arrow) (A) and dorsomedial thalamic nucleus (arrow) (B). Preoperative MRI T2* shows the SVS in the BA (arrow) (C). BA: basilar artery; DWI: diffusion weighted image; SVS: susceptibility vessel sign

NIHSS20, and the right pupil became dilated. When MRI was performed again, hyper-intense signals were noted on DWI from the ventral side of the midbrain to the cerebral peduncle and dorsomedial thalamic nucleus (Fig. 2A and 2B), which were not observed on admission. In addition, T2* findings suggesting susceptibility vessel sign (SVS) were noted at the end of the BA (Fig. 2C). As the affected area suggested infarction of the area supplied by the paramedian thalamic artery (PTA) due to basilar tip occlusion, emergency thrombectomy was carried out in order to improve the severe symptoms.

Under local anesthesia, an 8-Fr short sheath catheter (Terumo, Tokyo, Japan) was inserted via the right femoral artery. When angiography was performed by guiding an 8-Fr Fubuki ST $90 \mathrm{~cm}$ (Asahi Intecc, Aichi, Japan) to the left vertebral artery, the left P1 was showed, but a filling defect was noted from the tip of the BA to the right P1, and an influx of the contrast medium was observed slightly distally to the filling defect (Fig. 3A), indicating continuation of thrombectomy. Using the Penumbra system 5MAX ACE (Penumbra, Alameda, CA, USA) and a Marksman microcatheter (Medtronic, Irvine, CA, USA) coaxially, the Marksman was guided distally to the site of occlusion with an ASAHI CHIKAI black $200 \mathrm{~cm}$ (Asahi Intecc), and a Trevo XP Provue Retriever $4 \times 20 \mathrm{~cm}$ (Stryker, Minneapolis, MN, USA) was deployed. After confirming immediate flow restoration, the Penumbra system 5MAX ACE was guided proximally to the site of occlusion and retrieved by the combined technique. Grade 3 recanalization by the Thrombolysis In Cerebral Infarction scale was achieved by a first pass (Fig. 3B). The puncture-to-recanalization time was 23 minutes. After surgery, consciousness disturbance and anisocoria resolved, the NIHSS score improved to 5 , and the right P1 to the peripheral vessels was showed on MRA (Fig. 4). On the 3rd hospital day, atrial fibrillation was noted on the monitor and a diagnosis of cardiogenic cerebral embolism was made. The patient was discharged on the 32nd hospital day while continuing rehabilitation. 


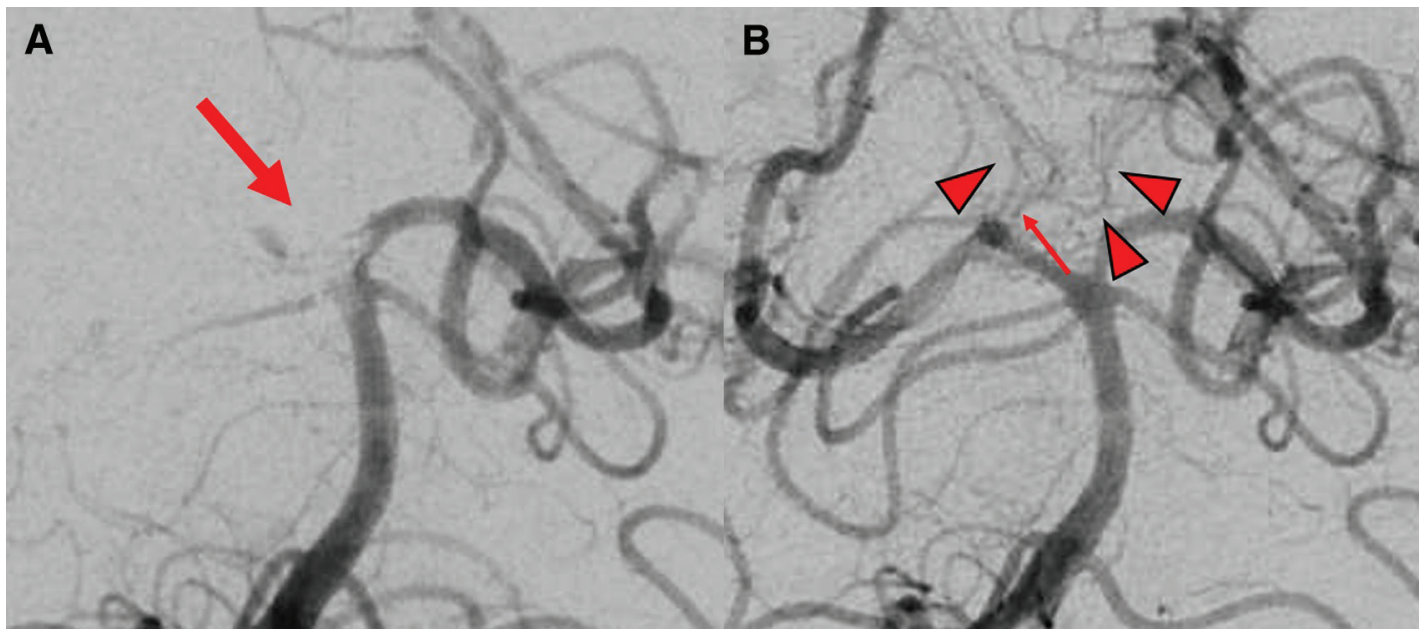

Fig. 3 DSA of the right vertebral artery. (A) DSA shows the thrombus from the top of the BA to the right P1 segment (arrow). (B) Postoperative DSA shows the perforator branch (arrow) from the right P1 segment, which is the common trunk for the bilateral PTA (arrowheads). BA: basilar artery; PTA: paramedian thalamic arteries

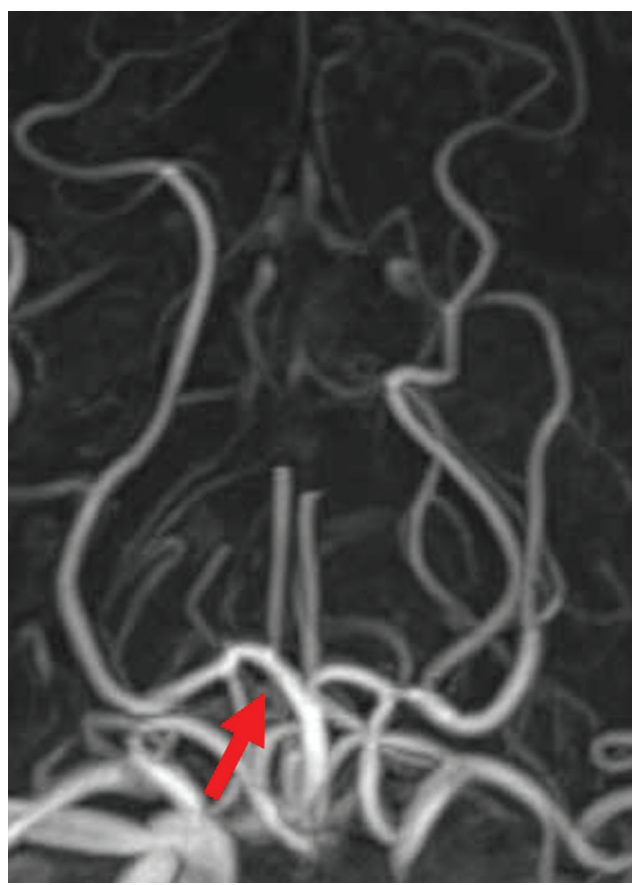

Fig. 4 Postoperative MRA. MRA shows recanalization of the right $\mathrm{P} 1$ segment (arrow).

The modified Rankin Scale (mRS) score was 1 at discharge and 0 after 3 months.

\section{Discussion}

In the patient presented here, thrombectomy for infarction of the midbrain and thalamus due to right P1 occlusion resulted in recanalization of the perforator branch, resulting in a favorable outcome. Kleine et al. ${ }^{5)}$ reported the occlusion of the lenticulostriate artery (LSA) induced by middle cerebral artery occlusion, which was correlated with infarct areas of the caudate nucleus, putamen, globus pallidus, and internal capsule supplied by these vessels. In this case, however, infarction was considered to have developed in the area supplied by a perforator that branched from the $\mathrm{P} 1$ as an embolus was trapped in the P1.

In our patient, who exhibited anisocoria and left hemiparesis, imaging findings suggested Weber's syndrome due to impairment of the ventromedial side of the midbrain, the condition was considered to have been caused by impairment of the cerebral peduncle and the root of oculomotor nerve, and the PTA, which is a perforator from the P1, was considered to have been the culprit vessel. ${ }^{6}$ In our patient, only the right P1 was occluded and the left P1 was patent, but examination of the tip of the BA after recanalization demonstrated marked improvement in delineation of the perforator branch (Fig. 3B). Percheron et al. ${ }^{7)}$ reported that, although infrequently, one artery originates from a unilateral posterior cerebral artery as a common vessel, and subsequently divides into left and right perforating arteries, and the common vessel is called the AOP (Fig. 5). The AOP is reportedly present at a rate of $4-12 \%$, and its occlusion causes a wide variety of symptoms, including consciousness disturbance, memory impairment, motor paralysis, aphasia, ataxia, and pupillary abnormalities. ${ }^{8)}$ Which symptoms occur depends on the area of infarction caused by occlusion of the AOP, and although the prognosis varies, the outcome is likely to be poor when the midbrain is included in the infarct area. ${ }^{8)}$ In our patient, PTA suggestive 


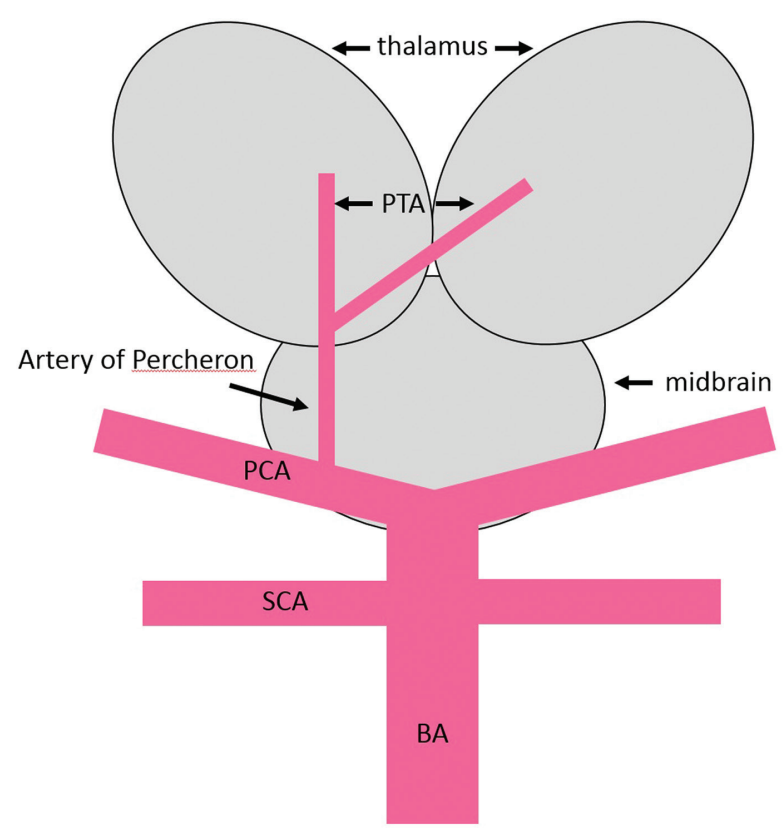

Fig. 5 The schema shows the AOP branching as a single common trunk for the bilateral paramedian thalamic arteries at the right P1 segment, which supplies blood to bilateral thalami. AOP: artery of Percheron; BA: basilar artery; PCA: posterior cerebral artery; PTA: paramedian thalamic artery; SCA: superior cerebellar artery

of blood supply from the right $\mathrm{P} 1$ to the opposite side was noted (Fig. 3B, arrow), and consciousness disturbance was considered to have been caused by occlusion of the bilateral PTAs due to occlusion of the AOP branching from the right P1. Furthermore, infarction of only the right thalamus was noted on MRI, but as consciousness deteriorated over time, ischemic injury of the left thalamus that was not detected on DWI was suspected. Infarction due to occlusion of the AOP is considered to occur in $4-18 \%$ of all patients with thalamic infarction, ${ }^{9)}$ but although there have been few case reports of infarction due to AOP occlusion, ${ }^{10-13)}$ there was no report of its treatment by thrombectomy according to our review of the literature. In neurological practice, it must be remembered that patients with occlusion of the P1 alone may exhibit marked neurological deficits, such as those observed in our patient, due to AOP occlusion.

In the present case, a definitive diagnosis of cerebral infarction due to LVO was unable to be made at the initial examination and was delayed until reexamination necessitated by gradual exacerbation of consciousness disturbance and left hemiparesis, resulting in prolongation of the time from occlusion to recanalization. However, as no hyperintense signals suggestive of acute-phase infarction were noted on DWI at the time of admission, despite the detection of hyper-intense signals on DWI at reexamination after worsening of symptoms, we judged the condition to not be irreversible and performed thrombectomy. Weber et al. ${ }^{14)}$ reported that therapeutic response can be obtained in the posterior circulation even by delayed treatment after the onset, unlike in the anterior circulation. However, as the perforator branch is a blind-ending artery without collaterals, unlike cortical vessels, and are vulnerable to ischemia, the necessity of prompt reperfusion should always be remembered.5)

In this patient, thrombectomy was performed using a stent retriever, but when stents are used, there is a risk of pull-out injury of perforators caused by strong traction of blood vessels. ${ }^{15,16)}$ As the areas supplied by perforators may be damaged by the procedure aimed at recanalization, a direct aspiration first pass technique (ADAPT) using a thrombus aspiration catheter was also considered for treatment. However, there have been reports that the first pass effect (FPE) is more likely to be obtained by the combined technique using a stent retriever and an aspiration catheter. ${ }^{17,18)}$ In addition, Bernava et al. ${ }^{19)}$ reported that recanalization is difficult to achieve by ADAPT depending on the angle of the vessel. In this patient, a thrombus aspiration catheter was guided in expectation of ADAPT, but thrombectomy using a stent retriever was judged to be preferable because of the angle from the BA to the P1, and recanalization was achieved after the first pass by thrombectomy using the combined technique. Even for infarction of an area supplied by a perforator branch, selection of the most effective device for obtaining FPE is considered important in conducting thrombectomy for LVO.

In some cases with perforator infarction of the posterior circulation, such as this case, recanalization of the perforator may be possible by retrieving the embolus in the large vessel, and some cases of severe perforator infarction are considered to be possible indications for thrombectomy of the large vessel.

\section{Conclusion}

In a patient who presented with consciousness disturbance and Weber's syndrome due to occlusion of the AOP, a favorable outcome was obtained by performing thrombectomy for occlusion of the posterior cerebral artery (P1 segment). Thrombectomy of a large vessel can also be effective in some patients with perforator infarction of the posterior circulation.

\section{Disclosure Statement}

The authors declare no conflict of interest. 


\section{References}

1) Nogueira RG, Jadhav AP, Haussen DC, et al: Thrombectomy 6 to 24 hours after stroke with a mismatch between deficit and infarct. N Engl J Med 2018; 378: 11-21.

2) Albers GW, Marks MP, Kemp S, et al: Thrombectomy for stroke at 6 to 16 hours with selection by perfusion imaging. N Engl J Med 2018; 378: 708-718.

3) van Houwelingen RC, Luijckx GJ, Mazuri A, et al: Safety and outcome of intra-arterial treatment for basilar artery occlusion. JAMA Neurol 2016; 73: 1225-1230.

4) Wang J, Qian J, Fan L, et al: Efficacy and safety of mechanical thrombectomy for M2 segment of middle cerebral artery: a systematic review and meta-analysis. J Neurol 2020 Jan 22. doi: 10.1007/s00415-020-09710-w. [Epub ahead of print]

5) Kleine JF, Beller E, Zimmer C, et al: Lenticulostriate infarctions after successful mechanical thrombectomy in middle cerebral artery occlusion. J Neurointerv Surg 2017; 9: 234-239.

6) Castaigne P, Lhermitte F, Buge A, et al: Paramedian thalamic and midbrain infarct: clinical and neuropathological study. Ann Neurol 1981; 10: 127-148.

7) Percheron G: Arteries of the human thalamus. II. Arteries and paramedian thalamic territory of the communicating basilar artery. Rev Neurol (Paris) 1976; 132: 309-324.

8) Kichloo A, Jamal SM, Zain EA, et al: Artery of percheron infarction: a short review. J Investig Med High Impact Case Rep 2019; 7: 2324709619867355.

9) Caruso P, Manganotti P, Moretti R: Complex neurological symptoms in bilateral thalamic stroke due to Percheron artery occlusion. Vasc Health Risk Manag 2017; 13: 11-14.

10) Kostanian V, Cramer SC: Artery of Percheron thrombolysis. AJNR Am J Neuroradiol 2007; 28: 870-871.
11) Liu J, Xiao H, Hu Z: Percheron infarction: is it just a rare cerebrovascular variant or a forewarning of severe multiple posterior circulation infarcts. J Stroke Cerebrovasc Dis 2019; 28: e27-e29.

12) Lazzaro NA, Wright B, Castillo $M$, et al: Artery of percheron infarction: imaging patterns and clinical spectrum. AJNR Am J Neuroradiol 2010; 31: 1283-1289.

13) Sandvig A, Lundberg S, Neuwirth J: Artery of Percheron infarction: a case report. J Med Case Rep 2017; 11: 221.

14) Weber R, Minnerup J, Nordmeyer H, et al: Thrombectomy in posterior circulation stroke: differences in procedures and outcome compared to anterior circulation stroke in the prospective multicentre REVASK registry. Eur J Neurol 2019; 26: 299-305.

15) Yoon W, Jung MY, Jung SH, et al: Subarachnoid hemorrhage in a multimodal approach heavily weighted toward mechanical thrombectomy with solitaire stent in acute stroke. Stroke 2013; 44: 414-419.

16) Prothmann $S$, Friedrich B, Boeckh-Behrens T, et al: Aspiration thrombectomy in clinical routine interventional stroke treatment : Is this the end of the stent retriever era? Clin Neuroradiol 2018; 28: 217-224.

17) Maegerlein C, Berndt MT, Mönch S, et al: Further development of combined techniques using stent retrievers, aspiration catheters and BGC: The PROTECTPLUS technique. Clin Neuroradiol 2020; 30: 59-65.

18) Maus V, Henkel S, Riabikin A, et al: The SAVE technique: large-scale experience for treatment of intracranial large vessel occlusions. Clin Neuroradiol 2019; 29: 669-676.

19) Bernava G, Rosi A, Boto J, et al: Direct thromboaspiration efficacy for mechanical thrombectomy is related to the angle of interaction between the aspiration catheter and the clot. J Neurointerv Surg 2020; 12: 396-400. 